\title{
Multimodal Peri-articular Injection with Tranexamic Acid can reduce postoperative blood loss versus Intravenous Tranexamic Acid in Total Knee Arthroplasty: A Randomized Controlled Trial
}

\author{
Hui-ming Peng, Wei Wang, Jin Lin*, Xi-sheng Weng, Wen-wei Qian and Wen-da Wang
}

\begin{abstract}
Background: Tranexamic acid (TXA) has shown significant reductions in blood loss and transfusion rates in total knee arthroplasty (TKA). However, the optimal administration route continues to be debated. The aim of this trial was to compare the effectiveness of intravenous (IV) versus peri-articular injection (PAl) application of tranexamic acid in patients undergoing total knee arthroplasty.
\end{abstract}

Methods: We conducted a randomized controlled, double-blinded study. A total of 93 patients undergoing primary unilateral TKA were randomly distributed between 2 groups: the IV group (47 cases; 1 g TXA IV) and the PAl group (46 cases; $1 \mathrm{~g}$ TXA injected peri-articularly). The amount of total and hidden blood loss (HBL), drainage, transfusion rate, hemoglobin and hematocrit drift, and complications were recorded.

Results: Peri-articular injection of TXA reduced total blood loss $(P<0.001)$ and HBL more than IV use of TXA $(P<$ 0.001). No patients in either group received a transfusion. No symptomatic deep venous thrombosis or other severe complications occurred.

Conclusion: Peri-articular injection of TXA significantly reduced total blood loss and hidden blood loss to a greater degree than IV injection in total knee arthroplasty without reduction of drainage volume.

Trial registration: Chinese Clinical Trial Registry, ChiCTR-INR-16010270. Date of registration: December 27, 2016.

Keywords: Peri-articular injection, Total knee arthroplasty, Tranexamic acid

\footnotetext{
*Correspondence: lin_chaos@sina.com

Department of Orthopaedic Surgery, Peking Union Medical College Hospital, Chinese Academy of Medical Sciences \& Peking Union Medical College, No.1,

Shuaifuyuan Wangfujing, Dongcheng District, Beijing 100730, China
}

(c) The Author(s). 2021 Open Access This article is licensed under a Creative Commons Attribution 4.0 International License, which permits use, sharing, adaptation, distribution and reproduction in any medium or format, as long as you give appropriate credit to the original author(s) and the source, provide a link to the Creative Commons licence, and indicate if changes were made. The images or other third party material in this article are included in the article's Creative Commons licence, unless indicated otherwise in a credit line to the material. If material is not included in the article's Creative Commons licence and your intended use is not permitted by statutory regulation or exceeds the permitted use, you will need to obtain permission directly from the copyright holder. To view a copy of this licence, visit http://creativecommons.org/licenses/by/4.0/. The Creative Commons Public Domain Dedication waiver (http://creativecommons.org/publicdomain/zero/1.0/) applies to the data made available in this article, unless otherwise stated in a credit line to the data. 


\section{Background}

Total knee arthroplasty (TKA) can result in an estimated 800 to $1200 \mathrm{ml}$ of blood loss, which has traditionally resulted in a transfusion rate as high as 53\% [1-3]. In addition, the requirement of a blood transfusion has been found to be an independent predictor of periprosthetic joint infection after TKA [4]. The management of blood loss during a TKA has been extensively studied [3,5-8], and one of the most promising agents that have emerged is tranexamic acid (TXA).

TXA is a competitive inhibitor of plasminogen activation that interferes with fibrinolysis improving hemostasis and helping prevent additional blood loss after tourniquet deflation during TKA [9]. This prothrombotic effect has raised concern about the risk of thromboembolic complications in total joint arthroplasty. This concern has led to the investigation of topical and local administration of TXA during TKA procedures with continued effectiveness in reducing blood loss and transfusion rates [10].

However, the ideal method of topical or local techniques varies widely from the simple soaking of the wound prior to joint closure to intra-articular injections after deep fascial closure. However, the limited exposure time of TXA with local tissues raises concerns about both simple soaking and intra-articular injections, especially in patients undergoing significant soft tissue releases. Peri-articular injections (PAI) of TXA eliminate the concern of limited local effect and have been shown to significantly improve blood loss and transfusion rates when compared to intra-articular injections [11].

Peri-articular injections aimed at multimodal analgesia are widely used in TKA and have proven to be more effective in providing analgesia compared to traditional methods $[12,13]$. To our knowledge, TXA has not been evaluated as a component of multimodal peri-articular injections. We hypothesized that adding TXA as a part of a peri-articular injection containing epinephrine would prolong the local effect of TXA directly on the injured tissue. This may result in reductions in blood loss and transfusion rates comparable to or better than intravenous administration of tranexamic acid but without the systemic toxicity of IV TXA.

Given the above, we evaluated the benefits and complications of an analgesic peri-articular injection with the addition of TXA to a standard IV TXA injection.

\section{Materials and methods}

\section{Study design}

This double-blinded randomized control trial was conducted from January 2017 to July 2018, and the study protocol was approved by our institutional review board and is registered in the Chinese Clinical Trial Registry (ChiCTR-INR-16010270). Written informed consent and research authorization for participation in this study were obtained from each patient before surgery.

Patients presenting during the study time frame undergoing elective unilateral, primary TKA for endstage osteoarthritis or rheumatoid arthritis were screened for inclusion into the study. Patients were then excluded for any of the following reasons: (1) an allergy to TXA; (2) preoperative hepatic or renal dysfunction; (3) serious cardiac or respiratory disease, including coronary artery stent placement; (4) congenital or acquired coagulopathy, as evidenced by an international normalized ratio (INR) of $>1.4$ or a partial thromboplastin time (PTT) of $>1.4$ times normal; (5) thrombocytopenia, as identified by a preoperative platelet count of $<150,000 /$ $\mathrm{mm}^{3}$; (6) a history of a pro-thrombotic condition; (7) pregnancy; (8) breastfeeding; (9) donated preoperative autologous blood; (10) an age of $<18$ years or $>80$ years; and/or (11) a preoperative hemoglobin level of $<10 \mathrm{~g} / \mathrm{dl}$.

Patients were then randomized to 1 of 2 groups: IV administration of TXA or PAI TXA, in accordance with the random number table. The randomization schedule was generated after the initiation of study enrollment (Fig. 1). The randomization was concealed by sealed, opaque envelopes and was only accessible to the nurse in the operating room who provided the IV and the injection material for each patient. This nurse was not included in data collection or analysis. The surgeons, patients, anesthesiologist, and the data collection team were blinded to randomization.

In the IV group, a 60-ml multimodal cocktail periarticular injection (MCPI) with ropivacaine, $200 \mathrm{mg} / 20$ $\mathrm{ml}$ (AstraZeneca AB, Sweden); morphine, $10 \mathrm{mg} / 1 \mathrm{ml}$ (domestic company); flurbiprofen axetil injection, $50 \mathrm{mg} /$ $5 \mathrm{ml}$ (Beijing Tide Pharmaceutical Co., Ltd., China); adrenaline, $0.25 \mathrm{mg}$ (1:1000); betamethasone, $7 \mathrm{mg} / 1 \mathrm{ml}$ (Schering-Plow Labo NV, Belgium); and $34 \mathrm{ml}$ of normal saline solution was prepared in three $20-\mathrm{ml}$ syringes. Ten minutes before skin incision, patients received 1000 $\mathrm{mg}$ of IV TXA (110 ml total volume) IV administration.

In the PAI group, the $60-\mathrm{ml}$ MCPI was the same as that in the IV TXA group, except that TXA $1000 \mathrm{mg} / 10$ ml (Guangzhou Baiyunshan Pharmaceutical Co., Ltd., China) was added. Before incision, patients received 110 $\mathrm{ml}$ of saline IV administration as a placebo.

\section{Surgical procedures and peri-operative care}

All patients underwent TKA under tourniquet control by a single surgeon (Jin Lin, MD). A medial parapatellar approach was utilized, and either a G-II PS (Smith \& Nephew, Memphis, USA) or Scorpio (Stryker, Limerick, Ireland) was inserted. No patella was resurfaced during the TKA procedure. The peri-articular injections were performed with the following technique: the first $20 \mathrm{ml}$ 


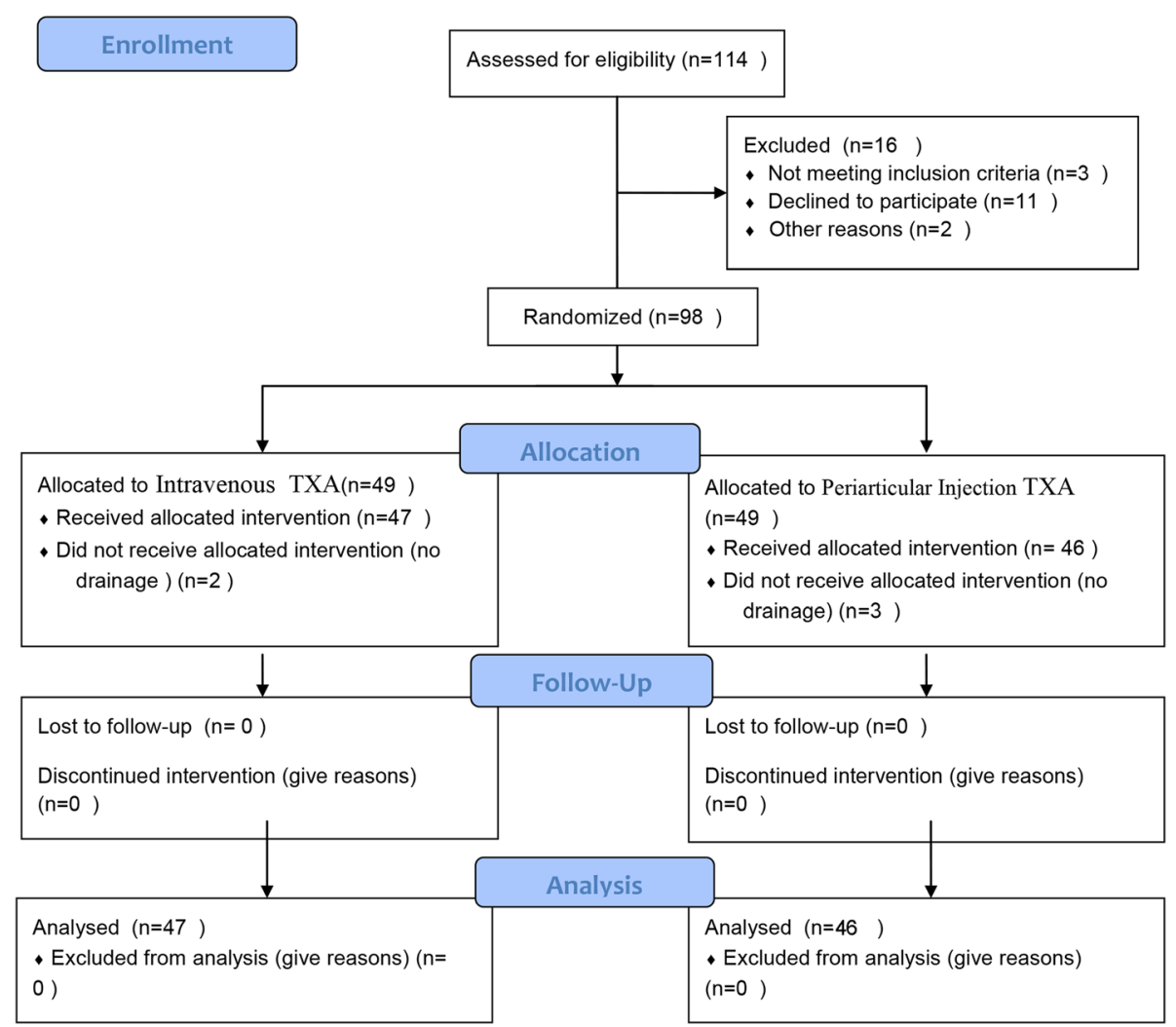

Fig. 1 CONSORT 2010 flow diagram

of the cocktail was injected into the posterior aspect of the capsule and structures of the knee joint immediately before implanting the prosthesis. After implantation, the remaining $40 \mathrm{ml}$ of the mixture was injected into the extensor mechanism, synovium, anterior capsule, pes anserinus, retinaculum, periosteum, iliotibial band, and collateral ligaments. A drain was placed within the lateral gutter in all patients prior to deep fascial closure.

Postoperatively, a vacuum wound drainage dressing was applied in every patient and a standardized rehabilitation protocol was implemented starting on day 1 . In both groups, the drains were removed at $48 \mathrm{~h}$, and the volume of fluid at 24 and $48 \mathrm{~h}$ was measured. Chemical and mechanical deep vein thrombosis (DVT) prophylaxis was instituted in all patients and consisted of thromboembolic deterrent stockings along with the daily subcutaneous injection of low molecular weight heparin $(0.1 \mathrm{ml} / 10 \mathrm{~kg}$ once a day) until discharge. The criteria for the transfusion of blood products included a hemoglobin level of $<8 \mathrm{~g} / \mathrm{dl}$ or a hemoglobin level of $<10 \mathrm{~g} / \mathrm{dl}$ in a patient with symptomatic anemia, or a patient deemed at high risk because of notable underlying cardiac comorbidities. After hospital discharge, patients were seen at 2 weeks, 4 weeks, and 12 weeks postoperatively. They were then seen twice a year or more frequently if complications had occurred.
The primary outcomes measured were the total blood loss (TBL), hidden blood loss (HBL), hemoglobin (HGB) and hematocrit (HCT) drift, and need for a blood transfusion. Hidden blood loss was calculated using the method described by Sehat et al. [14]. HGB and HCT drifts were defined as the difference between preoperative and postoperative minimum values.

Additionally, secondary outcomes included postoperative duplex ultrasounds at post-op days 3 and 14 to detect DVT. D-dimer levels were measured at $24 \mathrm{~h}$ postoperatively. Complications were measured up to 3 months postoperatively and included local soft tissue complications, skin necrosis, peroneal nerve palsies, superficial and deep surgical site infections, symptomatic VTEs, cerebrovascular accident, and myocardial infarction.

\section{Sample size}

The study design of this trial was an equivalence test, and the sample size was calculated based on the measured postoperative blood loss. We assumed an alpha error of 0.05 and applied an allocation ratio of 1 . A sample size of 37 participants, which allowed for a dropout rate of $10 \%$ (4 participants), was calculated to provide $80 \%$ power in detecting a difference of $150 \mathrm{ml}$ or reducing postoperative blood loss by $30 \%$ 
in favor of the PAI TXA (from 20 patients of our earlier pilot study); we considered these parameters to be clinically relevant [15].

\section{Statistical analysis}

The Statistical Package for Social Sciences (SPSS Inc, IBM, version 22) was used. For continuous variables, the mean and range were compared between the groups using Student $t$ tests. For categorical variables, data was summarized as the frequency and proportion. Proportions were compared between the groups using Fisher's exact test on univariate analysis.

\section{Results}

A total of 114 unilateral primary total knee arthroplasties were screened for eligibility to be included in the trial. Ninety-eight patients met inclusion criteria and were randomized into the study groups, with each group consisting of 49 patients. Of the 98 patients, five were lost to followup, leaving 47 patients in the IV TXA group and 46 patients in the PAI TXA group for statistical analysis. A participant flowchart is provided in Fig. 1.

The mean patient age was 68.2 years (range, 48 to 80 years), and the mean body mass index (BMI) was $27 \mathrm{~kg} /$ $\mathrm{m}^{2}$ (range, 19 to $38 \mathrm{~kg} / \mathrm{m}^{2}$ ). There were no significant differences between the 2 groups for baseline variables including age, sex, medical history, American Society of

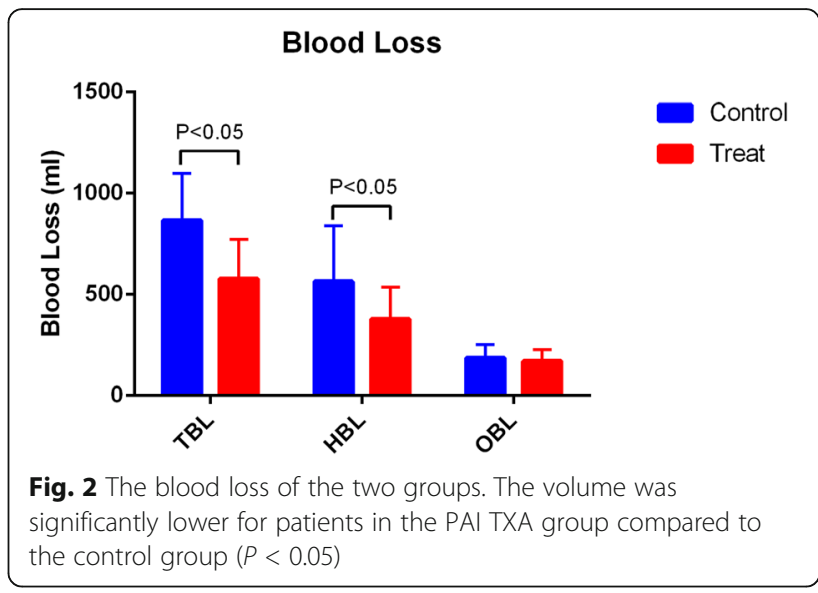

Anaesthesiologists (ASA) score, BMI, and preoperative laboratory values $(P>0.05)$ (Table 1$)$.

\section{Primary outcome}

Patients who received PAI TXA had significantly less TBL and HBL compared with patients who received IV TXA (TBL $641.6 \pm 234.0 \mathrm{ml}$ vs $896.0 \pm 248.6 \mathrm{ml}, P=$ 0.00 ; HBL $419.8 \pm 239.9 \mathrm{ml}$ vs $651.7 \pm 243.7 \mathrm{ml}, P=$ 0.00) (Fig. 2). The HCT drift on day 3 was significantly less in the PAI TXA group (Fig. 3). The first $24 \mathrm{~h}$ drainage post-operation, the total drainage volume, and the HGB drift on day 3 were not significantly different $(P>$

Table 1 Demographic data of the patients between two groups

\begin{tabular}{llll}
\hline Baseline characteristic & Control $(\boldsymbol{n = 4 7 )}$ & Treated $(\boldsymbol{n}=\mathbf{4 6})$ & $\boldsymbol{P}$ value \\
\hline Sex (male/female) & $6 / 41$ & $7 / 39$ & 0.733 \\
Age (years) & $68.13 \pm 8.12$ & $68.65 \pm 9.54$ & 0.776 \\
Height (cm) & $162.36 \pm 6.82$ & $659.87 \pm 6.91$ & 0.083 \\
Weight (kg) & $71.32 \pm 9.20$ & $26.65 \pm 12.20$ & 0.236 \\
BMI (kg/m $\left.{ }^{2}\right)$ & $27.06 \pm 3.19$ & $44 / 3$ & 0.749 \\
Diagnosis (OA/RA) & $44 / 2$ & $40.43 \%$ & 0.651 \\
Side (left) & $41.30 \%$ & $7 / 27 / 12$ & 0.931 \\
ASA score (1/2/3) & $5 / 21 / 21$ & $14.89 \%$ & 0.171 \\
Liver function abnormal (\%) & $19.57 \%$ & $10.64 \%$ & 0.551 \\
Pre-OP allergy (\%) & $15.22 \%$ & & 0.510 \\
Pre-OP laboratory values & & $130.82 \pm 10.35$ & \\
Pre-OP HGB & $132.32 \pm 10.25$ & $38.77 \pm 2.9296$ & 0.486 \\
Pre-OP HCT (\%) & $39.35 \pm 3.32$ & $1.19 \pm 1.70$ & 0.375 \\
Pre-OP D-dimer & $0.70 \pm 1.07$ & $2.95 \pm 0.69$ & 0.099 \\
Pre-OP Fig & $2.91 \pm 0.55$ & $5.64 \pm 1.33$ & 0.788 \\
Pre-OP WBC & $6.14 \pm 1.76$ & $4.27 \pm 0.33$ & 0.122 \\
Pre-OP RBC & $4.31 \pm 0.34$ & $238.07 \pm 73.58$ & 0.616 \\
Pre-OP PLT & $235.36 \pm 61.04$ & 0.847 \\
\hline
\end{tabular}

Notes: $B M I$ body mass index, OA osteoarthritis, RA rheumatoid arthritis, ASA American Society of Anaesthesiologists, HGB hemoglobin, $H C T$ hematocrit, Fig fibrinogen, $W B C$ white blood cell, RBC red blood cell, PLT platelet, OP operation 


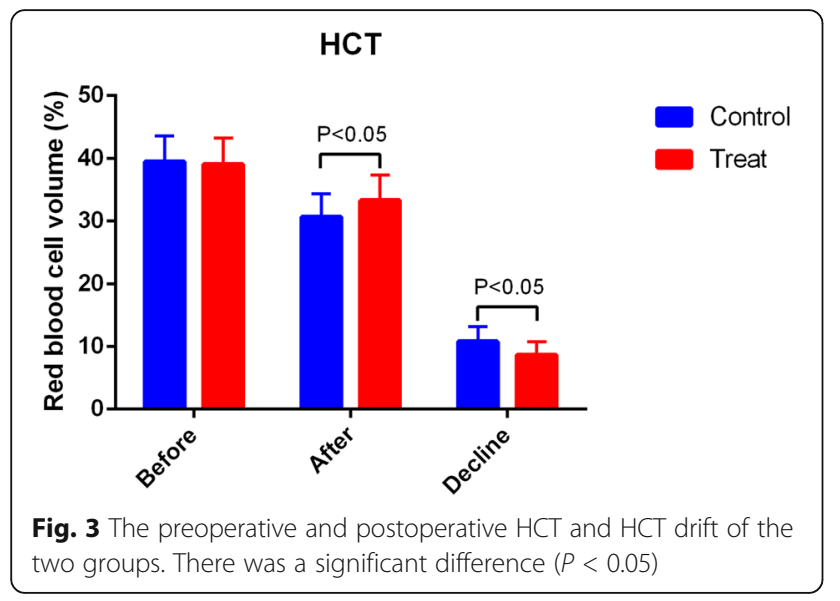

0.05) (Figs. 4 and 5). No patients required a blood transfusion (Table 2).

\section{Secondary outcome}

In the IV TXA cohort, 3 (6.3\%) of the patients sustained asymptomatic DVT compared with $2(4.3 \%)$ of the patients in the PAI TXA cohort, as confirmed by postoperative ultrasound $(P=0.32)$. The $D$-dimer values $24 \mathrm{~h}$ after operation were not significantly different $(P>0.05)$ (Table 3). None of the patients had clinical evidence of tense hemarthroses, subcutaneous hematomas, peroneal nerve palsies, surgical site infections, skin necrosis, symptomatic VTEs, cerebrovascular accident, myocardial infarction, or deep infection in the short-term (3 months) follow-up.

\section{Discussion}

Currently, multimodal cocktail peri-articular injections (MCPI) are widely used in peri-operative pain protocols $[12,13]$. This study found that the inclusion of TXA into these cocktails was equally efficacious as IV TXA in reducing postoperative blood loss and the need for

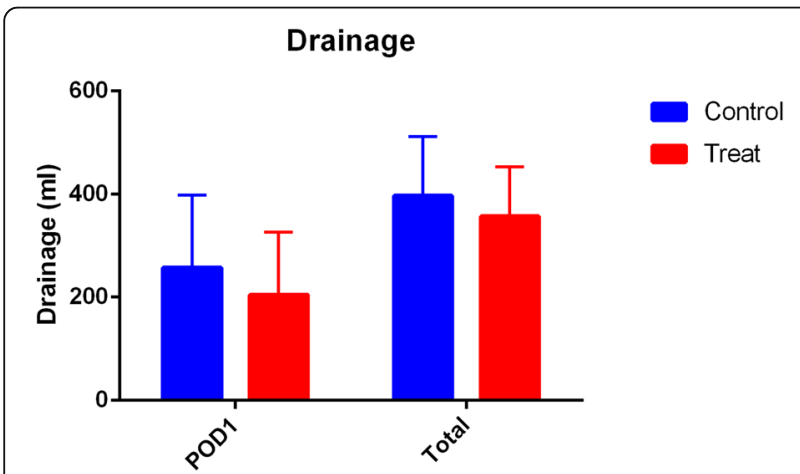

Fig. 4 The postoperative drainage volume of the two groups. The volume was lower for patients in the PAI TXA group compared to the control group but there was no significant difference $(P>0.05)$

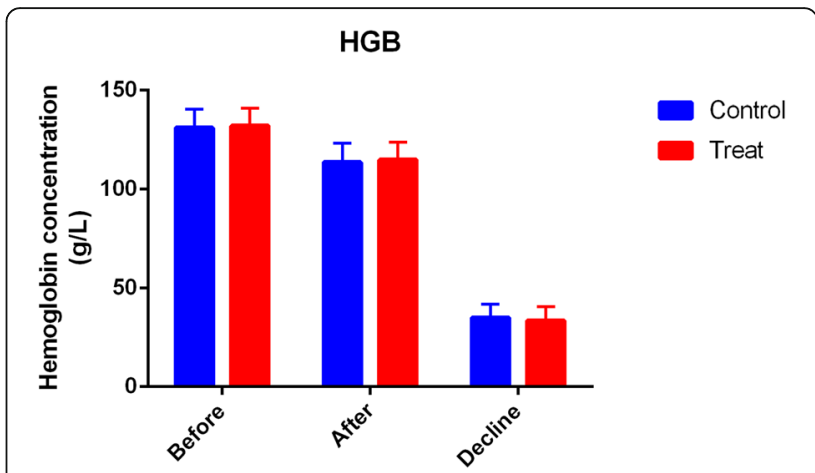

Fig. 5 The preoperative and postoperative $H G B$ and $H G B$ drift of the two groups. There was no significant difference $(P>0.05)$

transfusion. Furthermore, PAI TXA simplified the topical application of TXA and could potentially improve operative times as there is no need for TXA wound soaking. Therefore, with this technique of topical TXA administration, we gain an analgesic effect and reduce postoperative blood loss simultaneously.

The optimal dosage of TXA in peri-articular injections has yet to be determined. Mao et al. found $2000 \mathrm{mg}$ of TXA to be effective in reducing postoperative blood loss during TKA [16]. While Pinsornsak et al. showed a slight increase in the number of blood transfusions when using a 750-mg dose of TXA, which they attributed to a

Table 2 Primary outcomes of the patients between two groups

\begin{tabular}{|c|c|c|c|}
\hline Primary outcomes & Control $(n=47)$ & Treated $(n=46)$ & $P$ value \\
\hline TBL (ml) & $896.06 \pm 248.60$ & $641.64 \pm 234.02$ & 0.000 \\
\hline $\mathrm{HBL}(\mathrm{ml})$ & $651.74 \pm 243.74$ & $419.85 \pm 239.80$ & 0.000 \\
\hline DBL (ml) & $244.30 \pm 60.68$ & $221.79 \pm 59.03$ & 0.073 \\
\hline POD1 drainage (ml) & $255.11 \pm 139.82$ & $205.33 \pm 117.05$ & 0.066 \\
\hline Total drainage (ml) & $388.62 \pm 121.36$ & $343.59 \pm 118.06$ & 0.073 \\
\hline Tourniquet time (min) & $82.36 \pm 4.54$ & $82.93 \pm 3.21$ & 0.485 \\
\hline Anesthesia time (min) & $105.36 \pm 4.54$ & $104.30 \pm 3.52$ & 0.213 \\
\hline POD3 HGB & $106.76 \pm 13.78$ & $107.41 \pm 12.21$ & 0.811 \\
\hline HGB decline & $26.27 \pm 10.17$ & $24.09 \pm 9.63$ & 0.290 \\
\hline POD3 HCT (\%) & $30.24 \pm 3.65$ & $31.73 \pm 3.52$ & 0.048 \\
\hline HCT decline (\%) & $9.73 \pm 2.42$ & $7.39 \pm 2.80$ & 0.000 \\
\hline POD1 Fig & $2.98 \pm 0.596$ & $3.058 \pm 0.698$ & 0.540 \\
\hline Fig change & $0.0634 \pm 0.546$ & $0.111 \pm 0.524$ & 0.668 \\
\hline POD1 RBC & $3.81 \pm 0.41$ & $3.84 \pm 0.42$ & 0.719 \\
\hline POD3 RBC & $3.46 \pm 0.47$ & $3.51 \pm 0.42$ & 0.543 \\
\hline POD1 PLT & $202.09 \pm 53.87$ & $200.98 \pm 57.09$ & 0.924 \\
\hline POD3 PLT & $183.63 \pm 50.10$ & $182.74 \pm 49.36$ & 0.932 \\
\hline Transfusion (unit) & 0 & 0 & \\
\hline
\end{tabular}

Notes: $T B L$ total blood loss, $H B L$ hidden blood loss, $D B L$ dominant blood loss, $H G B$ hemoglobin, HCT hematocrit, Fig fibrinogen, RBC red blood cell, PLT platelet, $P O D$ post-operation day 
Table 3 Secondary outcomes of the patients between two groups

\begin{tabular}{llll}
\hline Secondary outcomes & Control $(\boldsymbol{n}=\mathbf{4 7})$ & Treated $(\boldsymbol{n}=\mathbf{4 6})$ & $\boldsymbol{P}$ value \\
\hline Post-OP DVT & $2 / 46$ & $3 / 47$ & 0.632 \\
Post-OP lower limb ecchymosis & $1 / 46$ & $7 / 47$ & 0.00 \\
POD1 D-dimer & $5.31 \pm 3.35$ & $4.41 \pm 2.55$ & 0.147 \\
D-dimer change & $4.61 \pm 3.53$ & $3.21 \pm 3.04$ & 0.043 \\
\hline
\end{tabular}

Notes: DVT deep vein thrombosis, $O P$ operation, $P O D$ post-operation day

lower preoperative hemoglobin level [17]. In our study, no patients required a blood transfusion while using a dose half of that used by Mao et al. We hypothesize that the use of epinephrine with TXA can provide similar protections while reducing the necessary dose and potentially systemic effects of peri-articular injections.

Good et al. [18] reported that the IV administration of TXA reduced external blood loss but not hidden blood loss after TKA. This is consistent with our results, which showed a significant difference in postoperative hidden blood loss in favor of PAI TXA. IV TXA is a systemic therapy and requires systemic distribution to exert its antibleeding effects, and only a small portion of intravenous TXA solution reaches the target tissue. PAI TXA can act directly on the injured tissue and for a longer duration. As we know that the efficiency of TXA depends on the timing and total dosage in use, in our study, the dosage of TXA is $1000 \mathrm{mg}$ in both groups, but PAI TXA significantly reduced blood loss than IV TXA in TKA, which would be related to the faster action, less waste, and longer action time of PAI TXA. Our results could be clinically significant in preventing blood transfusions, especially in those starting with lower preoperative hemoglobin levels. Unfortunately, due to the small sample size and no transfusions performed, this benefit was not detected in our study.

In this study, there were no symptomatic VTE in either group, and the results of asymptomatic DVT confirmed by ultrasound and D-dimer measurements revealed no significant difference between the two groups. Although there was no control group to determine the effects TXA may have had on VTE development, the latest meta-analysis supports the safety of TXA in joint arthroplasty [18].

We do acknowledge the limitations of our study. First, we did not assess functional factors such as VAS score, knee range of motion, knee swelling, and walking ability. Second, we did not measure serum levels of PAI TXA vs IV TXA, thus limiting our interpretation of its systemic effects. Third, this study excluded patients who would be deemed at the most risk if they were to receive IV TXA. To compare the safety of each modality, we would require a large number of patients and may not be practical to investigate in a prospective, randomized study. Fourth, the timing was also different with IV being given immediately preoperatively and PAI being injected in the later stages of the surgery, which may have made it difficult to identify the local effect of PAI TXA in reducing blood loss. In addition, our study did not involve the determination of the optimal dosage of TXA, which also is a shortage and needs further studies to compare whether different doses of TXA can produce different results.

This randomized controlled trial has demonstrated encouraging results regarding the novel use of TXA as part of a combined peri-articular injection in TKA with a reduction in blood loss versus the more commonly used IV administration of TXA. The use of locally injected TXA is an effective and simple method of reducing blood loss and should be considered as an alternative to IV or topical application in primary TKA.

\section{Abbreviations}

TXA: Tranexamic acid; TKA: Total knee arthroplasty; IV: Intravenous; PAl: Periarticular injection; IA: Intra-articular injection; TBL: Total blood loss; HBL: Hidden blood loss

\section{Acknowledgements \\ None}

\section{Authors' contributions}

Dr. HP, Dr. WW, and Dr. WW carried out the trial. Dr. HP and Dr. JL prepared the manuscript with support from Dr. XW and Dr. WQ. Dr. JL supervised the project. The authors read and approved the final manuscript.

\section{Funding}

2016 Perioperative Orthopeadic Blood Management Research Project from the China International Medical Exchange Foundation

\section{Declarations}

Ethics approval and consent to participate

The study protocol was approved by our institutional review board (Ethics Committee of Peking Union Medical College Hospital, Number: ZS1244) and is registered in the Chinese Clinical Trial Registry (Number: ChiCTR-INR16010270). Written informed consent and research authorization for participation in this study were obtained from each patient before surgery. IRB is in the attachments

Consent for publication

All the authors listed have approved the publication.

Competing interests

The authors declare that they have no competing interests. 
Received: 1 June 2021 Accepted: 20 August 2021

Published online: 03 September 2021

\section{References}

1. Cankaya D, Della Valle CJ. Blood loss and transfusion rates in the revision of unicompartmental knee arthroplasty to total knee arthroplasty are similar to those of primary total knee arthroplasty but are lower compared with the revision total knee arthroplasty. J Arthroplast. 2016;31(1):339-41. https://doi. org/10.1016/j.arth.2015.08.002.

2. Yang ZG, Chen WP, Wu LD. Effectiveness and safety of tranexamic acid in reducing blood loss in total knee arthroplasty: a meta-analysis. J Bone Joint Surg Am. 2012;94(13):1153-9. https://doi.org/10.2106/JBJS.K.00873.

3. Sizer SC, Cherian JJ, Elmallah RD, Pierce TP, Beaver WB, Mont MA. Predicting blood loss in total knee and hip arthroplasty. Orthop Clin North Am. 2015; 46(4):445-59. https://doi.org/10.1016/j.ocl.2015.06.002

4. Blanco JF, Díaz A, Melchor FR, da Casa C, Pescador D. Risk factors for periprosthetic joint infection after total knee arthroplasty. Arch Orthop Trauma Surg. 2020;140(2):239-45. https://doi.org/10.1007/s00402-019-03304-6.

5. Cip J, Widemschek M, Benesch T, Waibel R, Martin A. Does single use of an autologous transfusion system in TKA reduce the need for allogenic blood? a prospective randomized trial. Clin Orthop Relat Res. 2013;471(4):1319-25. https://doi.org/10.1007/s11999-012-2729-1.

6. Sabatini L, Trecci A, Imarisio D, Uslenghi MD, Bianco G, Scagnelli R. Fibrin tissue adhesive reduces postoperative blood loss in total knee arthroplasty. J Orthopaedics Ttraumatol. 2012;13(3):145-51. https://doi.org/10.1007/s101 95-012-0198-7.

7. Wang K, Ni S, Li Z, Zhong Q, Li R, Li H, et al. The effects of tourniquet use in total knee arthroplasty: a randomized, controlled trial. Knee Surg Sports Traumatol Arthroscopy. 2017;25(9):2849-57. https://doi.org/10.1007/s00167015-3964-2.

8. Fillingham YA, Ramkumar DB, Jevsevar DS, Yates AJ, Shores P, Mullen K, et al. The efficacy of tranexamic acid in total knee arthroplasty: a network meta-analysis. J Arthroplast. 2018;33(10):3090-8 e1.

9. Gillette BP, DeSimone LJ, Trousdale RT, Pagnano MW, Sierra RJ. Low risk of thromboembolic complications with tranexamic acid after primary total hip and knee arthroplasty. Clin Orthop Relat Res. 2013;471(1):150-4. https://doi. org/10.1007/s11999-012-2488-z.

10. Abdel MP, Chalmers BP, Taunton MJ, Pagnano MW, Trousdale RT, Sierra RJ, et al. Intravenous versus topical tranexamic acid in total knee arthroplasty: both effective in a randomized clinical trial of 640 patients. Jbjs. 2018; 100(12):1023-9. https://doi.org/10.2106/JBJS.17.00908.

11. Pinsornsak $P$, Rojanavijitkul S, Chumchuen S. Peri-articular tranexamic acid injection in total knee arthroplasty: a randomized controlled trial. BMC Musculoskelet Disord. 2016;17(1):313. https://doi.org/10.1186/s12891-016-1176-7.

12. Zhang LK, Ma JX, Kuang MJ, Ma XL. Comparison of periarticular local infiltration analgesia with femoral nerve block for total knee arthroplasty: a meta-analysis of randomized controlled trials. J Arthroplast. 2018;33(6):1972-8 e4.

13. Ross JA, Greenwood AC, Sasser P 3rd, Jiranek WA. Periarticular injections in knee and hip arthroplasty: where and what to inject. J Arthroplast. 2017; 32(9s):S77-s80.

14. Sehat KR, Evans RL, Newman JH. Hidden blood loss following hip and knee arthroplasty. Correct management of blood loss should take hidden loss into account. J Bone Jjoint Surg Br Vol. 2004;86(4):561-5.

15. Pinsornsak P, Chumchuen S. Can a modified Robert Jones bandage after knee arthroplasty reduce blood loss? A prospective randomized controlled trial. Clin Orthop Relat Res. 2013;471(5):1677-81. https://doi.org/10.1007/s11 999-013-2786-0.

16. Mao Z, Yue B, Wang Y, Yan M, Dai K. A comparative, retrospective study of periarticular and intra-articular injection of tranexamic acid for the management of postoperative blood loss after total knee arthroplasty. BMC Musculoskelet Disord. 2016:17(1):438. https://doi.org/10.1186/s12891-016-1293-3.

17. Good L, Peterson E, Lisander B. Tranexamic acid decreases external blood loss but not hidden blood loss in total knee replacement. $\mathrm{Br} J$ Anaesth. 2003;90(5):596-9. https://doi.org/10.1093/bja/aeg111.

18. Fillingham YA, Ramkumar DB, Jevsevar DS, Yates AJ, Shores P, Mullen K, et al. The safety of tranexamic acid in total joint arthroplasty: a direct metaanalysis. J Arthroplast. 2018;33(10):3070-82 e1.

\section{Publisher's Note}

Springer Nature remains neutral with regard to jurisdictional claims in published maps and institutional affiliations.

\section{Ready to submit your research? Choose BMC and benefit from}

- fast, convenient online submission

- thorough peer review by experienced researchers in your field

- rapid publication on acceptance

- support for research data, including large and complex data types

- gold Open Access which fosters wider collaboration and increased citations

- maximum visibility for your research: over $100 \mathrm{M}$ website views per year

At BMC, research is always in progress.

Learn more biomedcentral.com/submissions 